\title{
Comités de ética en investigación durante la epidemia del nuevo coronavirus (COVID-19) en Perú
}

\section{Research ethics committees during the novel coronavirus (COVID-19) epidemic in Peru}

Jorge A. García-Mostajo ${ }^{1, a}$, Violeta Alvarez-Arroyo ${ }^{1, b}$, Mariangela DuffoóRosales $^{1, \mathrm{~b}}$, Harold Jiménez-Arismendiz ${ }^{1, \mathrm{c}}$, Fulton P. Rivera ${ }^{2, \mathrm{~d}}$

\begin{abstract}
Señor editor,
La Organización Mundial de la Salud (OMS) declaró al actual brote de enfermedad por coronavirus (COVID-19) como una pandemia ${ }^{(1)}$, lo que llevó al gobierno del Perú a declarar el estado de emergencia sanitaria a nivel nacional y tomar una serie de medidas preventivas ${ }^{(2)}$. Las medidas adoptadas por distintos países en todo el mundo responden a la ausencia de tratamiento efectivo y una vacuna ante el COVID-19, las cuales aún se encuentran en investigación.
\end{abstract}

El desarrollo de investigación durante epidemias, especialmente en aquellas ocasionadas por agentes etiológicos nuevos o poco conocidos, es una medida urgente que debe formar parte de la respuesta a la emergencia sanitaria que estas situaciones representan ${ }^{(3)}$. Es así que, ante la reciente pandemia, la cantidad de publicaciones científicas sobre el tema se viene incrementando a gran velocidad ${ }^{(1)}$.

Sin embargo, las investigaciones realizadas durante la actual pandemia, no están exentas de revisión ética, ya que dada la situación de "crisis" podría existir un riesgo mayor de vulnerar principios éticos en investigación, incluyendo, entre otros, respeto a las personas, derecho a confidencialidad y privacidad de la información. Es ese sentido, la Organización Panamericana de la Salud (OPS) y la Red de América Latina y el Caribe de Comités Nacionales de Bioética UNESCO recomiendan realizar una revisión de todas las investigaciones donde participen seres humanos, permitiéndose una revisión ética acelerada, sin perder rigurosidad $^{(4,5)}$.

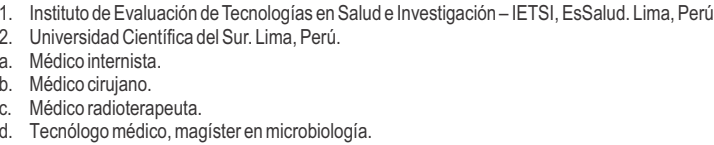

Así, lo que buscan las diferentes recomendaciones internacionales es garantizar la validez científica y los derechos y seguridad de los participantes en los estudios realizados durante los brotes, y es allí donde los Comités de Ética en Investigación (CEI) cumplen una función evaluadora y supervisora muy importante.

Los reglamentos y manuales de procedimientos de los CEI en el Perú, en general, no contemplan acciones específicas para la evaluación de estudios en situaciones de brotes y desastres y, más aún, luego las medidas dispuestas por el gobierno del Perú, como respuesta a la actual pandemia se limitaron las reuniones de los $\mathrm{CEI}$, habiendo retomado algunos de ellos sus sesiones de forma virtual.

Así, la actual pandemia representa un reto para todos los CEI a nivel nacional, siendo nuestra recomendación que los comités implementen mecanismos virtuales para la atención de solicitudes y revisión ética de los estudios, incluyendo el uso de plataformas que permitan reuniones para la deliberación; e incluyan entre sus procedimientos los de revisión expedita y exceptuación de revisión para estudios que no correspondan a ensayos clínicos, debiendo cada comité establecer las características de los estudios que pueden ser evaluados en cada una de las categorías mencionadas.

Por lo expuesto, existe una obligación moral de realizar investigaciones científicas oportunas, pero sin descuidar la protección de los sujetos que participen en ellas. Si bien es necesario agilizar los procesos para la revisión ética de los estudios, se debe garantizar el cumplimiento de los estándares éticos en investigación.

Conflictos de interés: Los autores niegan conflictos de interés.

Financiamiento: Autofinanciado. 
Contribuciones de los autores. JAGM, VAA, MDR, HJA y FPR participaron de la concepción del artículo, recolección de datos, su redacción y aprobación de la versión final

\section{REFERENCIAS BIBLIOGRÁFICAS}

1. World Health Organization. Global research on coronavirus disease ( COVID-19) [Internet]. 2020 [citado 1 de abril de 2020]. Disponible en: https://www. who.int/emergencies/diseases/no vel-coronavirus-2019/global-research-on-novelcoronavirus-2019-ncov

2. Ministerio de Salud. Decreto Supremo $\mathrm{N}^{\circ} 008$ 2020-SA [Internet]. Diario Oficial del Bicentenario El Peruano. 2020 [citado 1 de abril de 2020]. D i s p o n i ble

e $n$ : https://www.gob.pe/institucion/minsa/normaslegales/459902-008-2020-sa

3. Organización Panamericana de la Salud y Consejo de Organizaciones Internacionales de las Ciencias Médica (CIOMS). Pautas éticas internacionales para la investigación relacionada con la salud con seres humanos [Internet]. 2016 [citado 1 de abril de 2020]. Disponible en: https://cioms.ch/wpcontent / uploads / 2017 / 12/ CIOMS EthicalGuideline SP INTERIOR-FINAL.pdf

4. Pan American Health Organization / World Health Organization. Orientación ética sobre cuestiones planteadas por la pandemia del nuevo coronavirus (COVID-19) [Internet]. 2020 [citado 1 de abril de $\left.\begin{array}{llllll}2 & 0 & 2 & 0\end{array}\right]$ D is ponible e n : https://www.paho.org/es/documentos/orientac ion-etica-sobre-cuestiones-planteadas-porpandemia-nuevo-coronavirus-covid-19

5. La Red de América Latina y el Caribe de CNBUNESCO. Declaration on the Ethics of Biomedical Research in COVID-19 [Internet]. 2020 [citado 1 de abril de 2020]. Disponible en: https://en.unesco.org/news/ethics-researchtimes-pandemic-covid-19

6. Zhang H, Shao F, Gu J, Li L, Wang Y. Ethics Committee Reviews of Applications for Research Studies at 1 Hospital in China During the 2019 Novel Coronavirus Epidemic. JAMA [Internet]. 23 de marzo de 2020 [citado 1 de abril de 2020]; D i s p o n i b l e

e $\mathrm{n}$ : http://www.ncbi.nlm.nih.gov/pubmed/3220260 8. doi:10.1001/jama.2020.4362

\section{Correspondencia}

Harold S. Jiménez Arismendiz, MD

Dirección: Dirección de Investigación en Salud

Instituto de Evaluación de Tecnologías en Salud e Investigación Seguro Social de Salud - EsSalud. Av. Arenales 1302, oficina 310 - Jesús María, Lima, Perú

Teléfono: (51) (1) 265-6000 anexo 1955

Correo: dr.haroldjimenez@gmail.com

\section{Revisión de pares}

Recibido: 14/06/2020

Aceptado: 30/06/2020 